Case Report

\title{
Necrotizing Pseudomonas aeruginosa Community-Acquired Pneumonia: A Case Report and Review of the Literature
}

\author{
Satish Maharaj, ${ }^{1}$ Carmen Isache, ${ }^{2}$ Karan Seegobin, ${ }^{1}$ Simone Chang, ${ }^{3}$ and Grant Nelson ${ }^{1}$ \\ ${ }^{1}$ Department of Internal Medicine, University of Florida College of Medicine, Jacksonville, FL 32209, USA \\ ${ }^{2}$ Division of Infectious Diseases, University of Florida College of Medicine, Jacksonville, FL 32209, USA \\ ${ }^{3}$ University of Miami/Jackson Memorial Hospital, Miami, FL 33136, USA \\ Correspondence should be addressed to Satish Maharaj; satish.maharaj@jax.ufl.edu
}

Received 9 March 2017; Revised 19 April 2017; Accepted 26 April 2017; Published 17 May 2017

Academic Editor: Raul Colodner

Copyright (C) 2017 Satish Maharaj et al. This is an open access article distributed under the Creative Commons Attribution License, which permits unrestricted use, distribution, and reproduction in any medium, provided the original work is properly cited.

\begin{abstract}
Lung cavities are not typically associated with community-acquired pneumonia (CAP). CAP due to P. aeruginosa is rare and even less commonly causes necrotizing pneumonia. We report a case of $P$. aeruginosa CAP that progressed to necrotizing pneumonia and was eventually fatal. Procalcitonin (PCT) has been well investigated in guiding antibiotic therapy (especially CAP) in adults. In this case, PCT at presentation and sequentially was negative. We discuss this caveat and present hypotheses as to the sensitivity and specificity of PCT and C-reactive protein (CRP) in these patients. To better characterize P. aeruginosa CAP, we undertook a review of cases indexed in PubMed from 2001 to $2016(n=9)$. The data reveal that risk factors for P. aeruginosa CAP include smoking, alcohol use, obstructive lung disease, sinusitis, and hot tub use. The route of infection for $P$. aeruginosa CAP remains unknown. One of the most interesting findings on reviewing cases was that $P$. aeruginosa CAP involves the right upper lobe in the vast majority. We suggest that when physicians in the community see patients with distinctly upper lobe necrotizing or cavitary pneumonia, they should consider $P$. aeruginosa in their differential diagnosis. Further studies are needed to clarify route of infection, role of PCT and CRP, and optimal therapy including drug and duration.
\end{abstract}

\section{Introduction}

Necrotizing pneumonia refers to consolidation with multiple small cavities and parenchymal necrosis. Lung cavities have not typically been associated with community-acquired pneumonia (CAP). They develop when bacterial lung infections progress to necrosis despite optimal medical therapy. The pathogenesis of necrotizing pneumonia is not exactly known but is believed to be tissue necrosis from an exuberant inflammatory response driven by toxins produced by an invasive pathogen and/or associated vasculitis and venous thrombosis [1]. Pseudomonas aeruginosa is a Gram-negative bacterium that is notorious for severe hospital- acquired respiratory infections. Patients with serious pseudomonal infections often have risk factors such as being immunocompromised or on a ventilator [2]. However, CAP due to $P$. aeruginosa is rare [3] and has been infrequently known to cause necrotizing pneumonia.

\section{Case Presentation}

A 63-year-old female presented to her primary care physician with a 6-day history of fever, cough, and dyspnea. Her cough was productive of green sputum that was occasionally streaked with blood. She had mild chest discomfort but no chest pain or pleurisy at that time. Review of systems was otherwise unremarkable. Her medical history was significant for obstructive lung disease (COPD), hypothyroidism (well controlled), hypertension, and a long history of smoking (60 pack years). Two years ago, she had computed tomography (CT) scanning of the chest which showed emphysematous changes but no cavities. Despite having severe COPD, she continued to smoke daily and consume alcohol (80 units weekly). She used home nebulization for her COPD, generally utilizing sterile water, but occasionally tap water. There was no illicit drug use. She did not have any history of recent hospitalization within the preceding 90 days. 


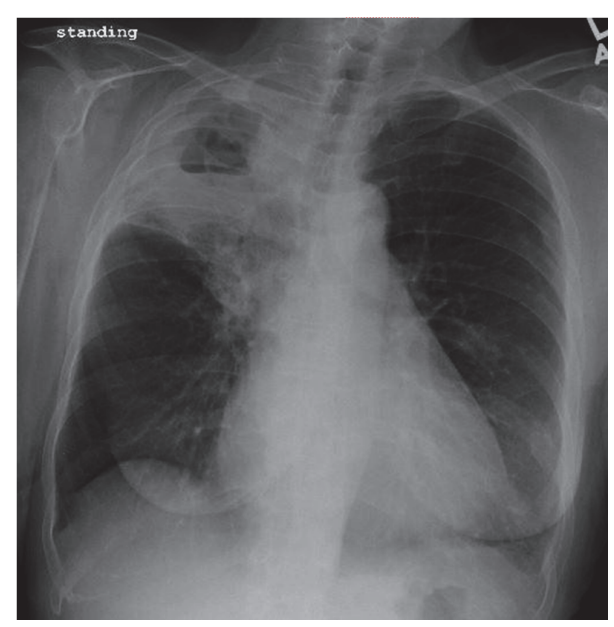

FIGURE 1: Chest X-ray with right upper lobe cavitating pneumonia.

On examination, the patient was not in any distress and auscultation revealed mild wheezing. She did not want to receive any further testing at that time and was prescribed a 5-day course of levofloxacin $750 \mathrm{mg}$ daily. Two weeks later, the patient presented for follow-up with worsening cough, increasing hemoptysis, and respiratory distress. On examination, she was in moderate respiratory distress with an oxygen saturation by pulse oximetry of $92 \%$ on room air, but not febrile or tachycardic, with a blood pressure of $167 / 97 \mathrm{mmHg}$.

Chest X-ray was done revealing right upper lobe opacification with a large air-fluid level suggestive of cavitating pneumonia (Figure 1). On admission then, leukocyte count was normal at $7.8 \times 10^{3} / \mathrm{mL}$ with no left shift; procalcitonin (PCT) was $0.12 \mathrm{ng} / \mathrm{mL}$ and C-reactive protein (CRP) was elevated at $143.8 \mathrm{mg} / \mathrm{L}$. Testing for renal, thyroid, and hepatic function and cardiac enzymes was within normal. Autoimmune panel was unremarkable. Testing for human immunodeficiency virus (HIV), bacterial and fungal urine, and serum antigens was all negative. CT scanning confirmed extensive opacification nearly completely filling the right upper lobe (Figure 2). Also new were associated cavitary regions within the right upper lobe. The largest area of cavitation measured $5.1 \times 4.2 \mathrm{~cm}$.

Two sputum cultures were collected separately and Gram stain revealed no squamous epithelial cells, few neutrophils, and Gram-negative rods. Within one day, both samples grew Pseudomonas aeruginosa, which was resistant to levofloxacin and ciprofloxacin. The patient was started on ceftazidime, as the isolate tested susceptible to cefepime, ceftazidime, gentamicin, piperacillin/tazobactam, and tobramycin. Two days into inpatient stay, the patient developed a leukocytosis of $13.1 \times 10^{3} / \mathrm{mL}$ with left shift. By that time, tuberculosis had been excluded with negative sputum for acid fast bacilli, along with a negative interferon-gamma release assay. Five days into admission, with no improvement, the patient underwent bronchoalveolar lavage and culture again grew $P$. aeruginosa with negative tuberculosis testing. Quantitative microbiologic analysis of the bronchoalveolar lavage was

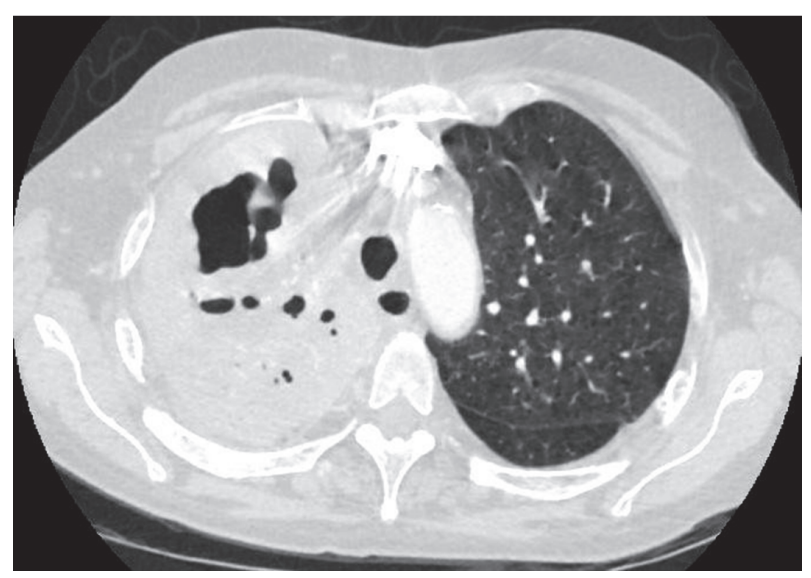

FIGURE 2: CT scan of the right upper lobe.

reported at 20,000 colonies $/ \mathrm{mL}$. The pattern of antibacterial susceptibility was exactly the same as that reported on the sputum cultures. One week into admission, the patient developed respiratory failure requiring intubation and was admitted to intensive care. Despite prolonged treatment, the patient died from progressive respiratory and multiorgan failure two weeks from admission.

\section{Discussion and Literature Review}

3.1. Pathophysiology. As this case demonstrates, P. aeruginosa CAP can progress to necrotizing pneumonia that can be fatal. Generally speaking, the progression from CAP to necrotizing pneumonia is usually rapidly progressive and these patients tend to present shortly after in acute respiratory distress. However, $P$. aeruginosa CAP appears to be an insidious disease, which can take weeks before respiratory decompensation. In the case above, we hypothesize that over the initial two weeks there would have been progressive necrosis, with coalescing of these foci into cavities which were seen on chest imaging (Figure 2). One similar case reported on pathologic findings from autopsy [4]; the affected lung showed infiltration by macrophages and neutrophils, with marked alveolar necrosis and hemorrhage. These findings can be explained as $P$. aeruginosa is invasive and can cause a thrombotic endarteritis [1].

3.2. Laboratory Investigations. CRP and more recently PCT have entered mainstream use in helping physicians distinguish between bacterial and nonbacterial causes of pneumonia. PCT is an acute-phase hormokine released by the parenchymal cells in patients with invasive bacterial infections [5]. PCT levels are also elevated in patients with bacteremia [6]. Research has confirmed that PCT is part of the complex innate immune response leading to an inflammatory state. Transcription and translation of PCT have been demonstrated both in organs and in macrophages as a result of bacterial infection and proinflammatory cytokine release. PCT has been well investigated in guiding antibiotic therapy by predicting the probability of a bacterial etiology of respiratory tract infection (especially CAP) in adults. A 
TABLE 1: Recent cases of $P$. aeruginosa CAP.

\begin{tabular}{|c|c|c|c|c|c|}
\hline Reference & $\begin{array}{l}\text { Age } \\
\text { (yrs) }\end{array}$ & Sex & Risk factors & $\begin{array}{l}\text { Location of } \\
\text { pneumonia }\end{array}$ & Treatment and outcome \\
\hline (1) Crnich et al. [14] & 40 & M & $\begin{array}{l}\text { Smoking, alcohol use, } \\
\text { emphysema, hot tub use }\end{array}$ & $\begin{array}{l}\text { Right upper and } \\
\text { middle lobes }\end{array}$ & $\begin{array}{l}\text { Amp, Cipro ( } 6 \text { weeks total); } \\
\text { recovery }\end{array}$ \\
\hline (2) Patel et al. [15] & 83 & $\mathrm{~F}$ & Asthma & Right upper lobe & $\begin{array}{l}\text { Ceft, Cipro, Tob, Pip ( } 34 \text { days } \\
\text { total); recovery }\end{array}$ \\
\hline (3) Huhulescu et al. [16] & 49 & $\mathrm{~F}$ & Smoking, hot tub use & Left lung & $\begin{array}{l}\text { Pip, Mox; multiorgan failure and } \\
\text { death }\end{array}$ \\
\hline (4) Okamoto et al. [17] & 39 & $\mathrm{~F}$ & Smoking, alcohol use & Right upper lobe & $\begin{array}{l}\text { Ceft, Cipro, Mero, steroids, } \\
\text { plasmapheresis; recovery }\end{array}$ \\
\hline (5) Takajo et al. [4] & 50 & $\mathrm{~F}$ & - & Right upper lobe & $\begin{array}{l}\text { Mero; respiratory failure and } \\
\text { death }\end{array}$ \\
\hline (6) Fujii et al. [18] & 29 & M & Sinusitis & Right upper lobe & Pip, Levo, Tob, Cipro; recovery \\
\hline (7) Kunimasa et al. [19] & 25 & M & Smoking & Right upper lobe & $\begin{array}{l}\text { Amp, Mero, Levo ( } 4 \text { weeks); } \\
\text { recovery }\end{array}$ \\
\hline (8) Gharabaghi et al. [20] & 26 & M & - & Left upper lobe & Oflox, Cipro (2 weeks); recovery \\
\hline (9) Present case & 63 & $\mathrm{~F}$ & $\begin{array}{l}\text { Smoking, alcohol use, } \\
\text { emphysema }\end{array}$ & Right upper lobe & $\begin{array}{l}\text { Levo, Ceftaz; multiorgan failure } \\
\text { and death }\end{array}$ \\
\hline
\end{tabular}

Amp, ampicillin; Ceft, ceftriaxone; Ceftaz, ceftazidime; Cipro, ciprofloxacin; Levo, levofloxacin; Mero, meropenem; Mox, moxifloxacin; Oflox, ofloxacin; Pip, piperacillin; Tob, tobramycin.

PCT cut-off of $0.50 \mathrm{ng} / \mathrm{mL}$ has been recommended to rule out bacterial infection in clinical practice [7]. Sequential PCT levels have been advocated for use in guiding antimicrobial therapy [5].

This data is interesting when applied to the case above. PCT was not elevated despite the presence of necrotizing pneumonia with clinical, radiologic, and microbiologic evidence of bacterial respiratory tract infection. Sequential PCT levels were also repeatedly negative $(<0.50 \mathrm{ng} / \mathrm{mL})$. One hypothesis is that the mechanisms of activating PCT transcription were not stimulated by localized $P$. aeruginosa necrotizing infection. The leukocyte count was surprisingly normal as well, also suggesting that the infection was relatively contained. Alternatively, the PCT cut-off may need to be lowered for detection in this population.

Studies reporting on PCT in patients with complicated parapneumonic effusions and empyema have yielded mixed results. Among 18 patients with culture-positive empyema, the sensitivity and specificity of PCT were $76 \%$ and $81 \%$, respectively [8]. This poor discriminatory performance was even using a lower threshold of $0.19 \mathrm{ng} / \mathrm{mL}$. In a prospective study, Doğan et al. compared 33 patients with parapneumonic effusions (including 22 empyemas) with controls (nonparapneumonic effusions). Using a cut-off level of $0.105 \mathrm{ng} / \mathrm{mL}$, the sensitivity and specificity of PCT were $67 \%$ and $97 \%$, respectively [9]. This case and the evidence from these studies demonstrate that PCT may remain low in localized or contained pulmonary infection, even when clinically and radiologically advanced. We suggest that despite being one of the best biomarkers for pulmonary infection, the discriminatory value of PCT in the diagnosis of localized necrotizing or cavitary pneumonia requires further investigation. We also hypothesize that the PCT cut-off of $0.50 \mathrm{ng} / \mathrm{mL}$ used to discriminate bacterial pneumonia may not be reliable in these cases to guide antibiotic therapy. Until this is clarified, a normal leukocyte count and low PCT should not falsely reassure the physician or patient as to the gravity of the illness.

These are of course issues which need to be evaluated in large scale studies. Whether these findings are dependent on bacterial etiology also remains to be investigated. Theories that may explain the low sensitivity of PCT include the absence of macrophage activation leading to TNF and the inability of the pleura to synthesize PCT. This second hypothesis deserves special mention as it has already been shown that PCT levels in pleural fluid from patients with empyema are of little discriminatory value [10].

CRP is an acute-phase protein that is synthesized by hepatocytes. It is widely available and often used to screen for infectious processes. It has been reported to be less sensitive than PCT for the detection of bacterial pneumonia and is well known to be nonspecific. However, in complicated pneumonia and localized infection, an elevated CRP level has been reported to have better discriminatory performance than PCT, in both the serum and pleural fluid [10-12], therefore, measuring CRP in cases where cavitary or necrotizing pneumonia is suspected to be of value.

3.3. Literature Review. Rare cases of CAP from P. aeruginosa have been reported since the 1960s. In what we found to be the most extensive review of $P$. aeruginosa CAP, Hatchette et al. analyzed all cases indexed in PubMed from 1966 to $2000(n=11)$ [13]. To better characterize $P$. aeruginosa CAP and assess for any evolution in the pattern of disease, we undertook a review of cases indexed in PubMed from 2001 to $2016(n=9)$. Cases were reviewed if they involved adults with no recent hospitalization, with positive identification of $P$. aeruginosa from either the sputum or blood, and clinical and radiologic confirmation of active pneumonia. These cases are summarized in Table 1. 
3.4. Risk Factors. On review of the nine cases reported in the last 15 years, patients affected continue to be middleaged with a mean age of $45 \pm 19$ years. There was no gender predisposition with five females and four males affected. Risk factors identified were smoking, alcohol use, and obstructive lung disease. Less common risk factors include sinusitis and hot tub use. The route of infection for $P$. aeruginosa CAP remains unknown. One case reported direct transmission between a mother and daughter [15], but in other cases there was no history of ill contacts. Several cases have documented exposure to contaminated aerosolized water [16, 21, 22]. It is well known that $P$. aeruginosa can contaminate even distilled water, persisting up to a year after inoculation in bottled water [23].

Our patient, along with other cases reported, had emphysematous lung changes. The pathophysiology behind P. aeruginosa CAP in patients with COPD is complex and remains to be elucidated. While early in emphysema there is a matched V/Q defect, over time hyperventilation develops and blood flow decreases, leading to V/Q mismatching. Combined with decreased mucociliary clearance from smoking, this can be favorable to growth of aerobic bacteria. It has been shown that a subset of patients with COPD, ranging from $4 \%$ to $15 \%$, are colonized with a clone of $P$. aeruginosa $[24,25]$. This has been likened to patterns of chronic infection resembling those observed in cystic fibrosis $[25,26]$. Persistent infections can lead to symptomatic exacerbations via clonal transformation to the mucoid $P$. aeruginosa phenotype. This phenotype was grown in one case of $P$. aeruginosa CAP [20]. However, this does not fully explain the mechanism of acute $P$. aeruginosa infection in the community with resulting inflammation and necrosis.

Patients generally presented with the nonspecific symptoms of cough and dyspnea, but some reported hemoptysis and chest pain $[4,17,19]$. Similar to what Hatchette et al. reported, on presentation there was a highly variable leukocyte count with cases reporting low, normal, and high levels. Perhaps reflecting the incorporation of CRP since Hatchette et al. did their review, 4 cases reported an elevated CRP ranging from 6.6 to $38.1 \mathrm{mg} / \mathrm{L}[16,17]$.

3.5. Location of Pneumonia. One of the most interesting findings reported by Hatchette et al. was that $P$. aeruginosa CAP involved the right upper lobe (RUL) in two-thirds of patients. In reviewing recent cases, this finding was reproduced with the vast majority (7/9) involving the RUL. Except one case which was not specified, all involved the upper lobes. The reason why $P$. aeruginosa CAP appears to have a predilection to involve the upper lobes has never been investigated. $P$. aeruginosa is of course an aerobic bacterium and it can be hypothesized that the upper lobes provide a more favorable environment by virtue of higher ventilation to perfusion ratios. We suggest this can be a clinical clue. When physicians in the community see patients with a distinctly upper lobe necrotizing or cavitary pneumonia they should consider $P$. aeruginosa in the differential diagnosis in the appropriate clinical scenario. A low index of suspicion can be beneficial as chest radiography may lag behind clinical status and early in the disease course necrotizing pneumonia can appear as consolidation only, leading to an underestimation of the degree of parenchymal destruction [1].

3.6. Prognosis and Complications. Out of the nine P. aeruginosa CAP cases recently reported, three were fatal highlighting the severity of this disease. Younger patients tended to do better. In fatal cases the disease course involved development of septic shock characterized by organ failure. Injury to the alveolar epithelium allows the release of proinflammatory mediators into the circulation that are primarily responsible for septic shock [27]. In cases of recovery, complications included parenchymal scarring and recrudescence requiring repeated antibiotic courses. This brings up the question of duration of treatment for $P$. aeruginosa CAP. In the cases reviewed with recovery, duration of therapy varied from 2 to 6 weeks.

\section{Conclusion}

Although CAP due to $P$. aeruginosa is rare, it can cause necrotizing pneumonia with high morbidity and mortality. Physicians must maintain a high level of suspicion as $P$. aeruginosa CAP can have an insidious course. Risk factors identified were smoking, alcohol use, and obstructive lung disease. Imaging can demonstrate coalescing of infectious foci into cavities and is recommended when there is clinical suspicion. However, chest radiography may lag behind clinical status. A review of the literature suggests that when physicians in the community see patients with a distinctly upper lobe pneumonia they should consider $P$. aeruginosa in their differential diagnosis. The biomarker PCT has been well investigated in guiding antibiotic therapy for CAP; however, we suggest that based on current evidence, PCT use in this way is not reliable in management of localized necrotizing or cavitary pneumonia. CRP appears to be more sensitive in these cases. The duration of treatment has not been well defined but a minimum of 2 weeks is reasonable. Further studies are needed to clarify the route of infection, role of PCT and CRP, and optimal therapy including drug and duration.

\section{Conflicts of Interest}

The authors declare that they have no conflicts of interest.

\section{References}

[1] Y.-F. Tsai and Y.-H. Ku, "Necrotizing pneumonia: A rare complication of pneumonia requiring special consideration," Current Opinion in Pulmonary Medicine, vol. 18, no. 3, pp. 246-252, 2012.

[2] J. Rello, B. Borgatta, and T. Lisboa, "Risk factors for pseudomonas aeruginosa pneumonia in the early twenty-first century," Intensive Care Medicine, vol. 39, no. 12, pp. 2204-2206, 2013.

[3] J. Almirall, I. Bolibar, J. Vidal et al., "Epidemiology of community-acquired pneumonia in adults: a population-based study," European Respiratory Journal, vol. 15, pp. 757-763, 2000.

[4] D. Takajo, K. Iwaya, Y. Katsurada et al., "Community-acquired lobar pneumonia caused by Pseudomonas aeruginosa infection 
in Japan: A case report with histological and immunohistochemical examination," Pathology International, vol. 64, no. 5, pp. 224-230, 2014.

[5] D. N. Gilbert, "Procalcitonin as a biomarker in respiratory tract infection," Clinical Infectious Diseases, vol. 52, supplement 4, pp. S346-S350, 2011.

[6] K. L. Becker, R. Snider, and E. S. Nylen, "Procalcitonin assay in systemic inflammation, infection, and sepsis: clinical utility and limitations," Critical Care Medicine, vol. 36, no. 3, pp. 941-952, 2008.

[7] M. Christ-Crain and B. Müller, "Biomarkers in respiratory tract infections: diagnostic guides to antibiotic prescription, prognostic markers and mediators," European Respiratory Journal, vol. 30, no. 3, pp. 556-573, 2007.

[8] M.-C. Lin, Y.-C. Chen, J.-T. Wu, Y.-C. Ko, and C.-C. Wang, "Diagnostic and prognostic values of pleural fluid procalcitonin in parapneumonic pleural effusions," Chest, vol. 136, no. 1, pp. 205-211, 2009.

[9] C. Doğan, S. Bilaçeroğlu, A. K. Çirak, A. Özsöz, and D. Özbek, "Diagnostic value of pleural fluid and serum procalcitonin levels in the diagnosis of parapneumonic pleural effusion," Tüberküloz ve Toraks, vol. 61, no. 2, pp. 103-109, 2013.

[10] J. M. Porcel, M. Vives, G. Cao et al., "Biomarkers of infection for the differential diagnosis of pleural effusions," European Respiratory Journal, vol. 34, no. 6, pp. 1383-1389, 2009.

[11] S. C. Chen, W. Chen, W. H. Hsu, Y. H. Yu, and C. M. Shih, "Role of pleural fluid C-reactive protein concentration in discriminating uncomplicated parapneumonic pleural effusions from complicated parapneumonic effusion and empyema," Lung, vol. 184, no. 3, pp. 141-145, 2006.

[12] L. Coelho, P. Povoa, E. Almeida et al., "Usefulness of C-reactive protein in monitoring the severe community-acquired pneumonia clinical course," Critical Care, vol. 11, article R92, 2007.

[13] T. F. Hatchette, R. Gupta, and T. J. Marrie, "Pseudomonas aeruginosa community-acquired pneumonia in previously healthy adults: Case report and review of the literature," Clinical Infectious Diseases, vol. 31, no. 6, pp. 1349-1356, 2000.

[14] C. J. Crnich, B. Gordon, and D. Andes, "Hot tub-associated necrotizing pneumonia due to pseudomonas aeruginosa," Clinical Infectious Diseases, vol. 36, no. 3, pp. e55-e57, 2003, Feb 1.

[15] P. Patel, S. Whittier, and E. Frank, "Person-to-person transmission of Pseudomonas pneumonia in the community: Documentation by pulsed-field electrophoresis," Southern Medical Journal, vol. 95, no. 6, pp. 653-656, 2002.

[16] S. Huhulescu, M. Simon, M. Lubnow et al., "Fatal Pseudomonas aeruginosa pneumonia in a previously healthy woman was most likely associated with a contaminated hot tub," Infection, vol. 39, no. 3, pp. 265-269, 2011.

[17] M. Okamoto, J. Yamaoka, S. Chikayama et al., "Successful treatment of a previously healthy woman with Pseudomonas aeruginosa community-acquired pneumonia with plasmapheresis," Internal Medicine, vol. 51, no. 19, pp. 2809-2812, 2012.

[18] A. Fujii, M. Seki, M. Higashiguchi et al., "Community-acquired, hospital-acquired, and healthcare-associated pneumonia caused by Pseudomonas aeruginosa," Respiratory Medicine Case Reports, vol. 12, pp. 30-33, 2014.

[19] K. Kunimasa, T. Ishida, S. Kimura et al., "Successful treatment of fulminant community-acquired Pseudomonas aeruginosa necrotizing pneumonia in a previously healthy young man," Internal Medicine, vol. 51, no. 17, pp. 2473-2478, 2012.
[20] M. A. Gharabaghi, S. M. Abdollahi, E. Safavi et al., "Community acquired pseudomonas pneumonia in an immune competent host," BMJ Case Reports, vol. 2012, 2012, May 26.

[21] H. D. Rose, T. R. Franson, N. K. Sheth, M. J. Chusid, A. M. Macher, and C. H. Zeirdt, "Pseudomonas Pneumonia Associated With Use of a Home Whirlpool Spa," JAMA: The Journal of the American Medical Association, vol. 250, no. 15, pp. 20272029, 1983.

[22] A. A. Harris, L. Goodman, and S. Levin, "Community-acquired Pseudomonas aeruginosa pneumonia associated with the use of a home humidifier," The Western Journal of Medicine, vol. 141, no. 4, pp. 521-523, 1984.

[23] D. W. Warburton, B. Bowen, and A. Konkle, "The survival and recovery of pseudomonas aeruginosa and its effect upon salmonellae in water: methodology to test bottled water in canada," Canadian Journal of Microbiology, vol. 40, no. 12, pp. 987-992, 1994.

[24] I. S. Patel, T. A. Seemungal, M. Wilks et al., "Relationship between bacterial colonisation and the frequency, character, and severity of COPD exacerbations," Thorax, vol. 57, pp. 759$764,2002$.

[25] L. Martínez-Solano, M. D. Macia, A. Fajardo, A. Oliver, and J. L. Martinez, "Chronic Pseudomonas aeruginosa infection in chronic obstructive pulmonary disease," Clinical Infectious Diseases, vol. 47, no. 12, pp. 1526-1533, 2008.

[26] S. L. Gellatly and R. E. W. Hancock, "Pseudomonas aeruginosa: new insights into pathogenesis and host defenses," Pathogens and Disease, vol. 67, no. 3, pp. 159-173, 2013.

[27] K. Kurahashi, O. Kajikawa, T. Sawa et al., "Pathogenesis of septic shock in Pseudomonas aeruginosa pneumonia," Journal of Clinical Investigation, vol. 104, no. 6, pp. 743-750, 1999. 


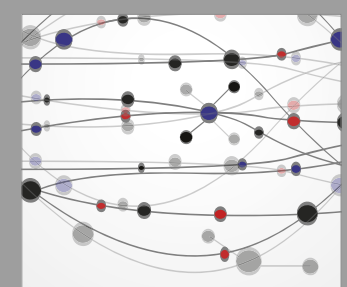

The Scientific World Journal
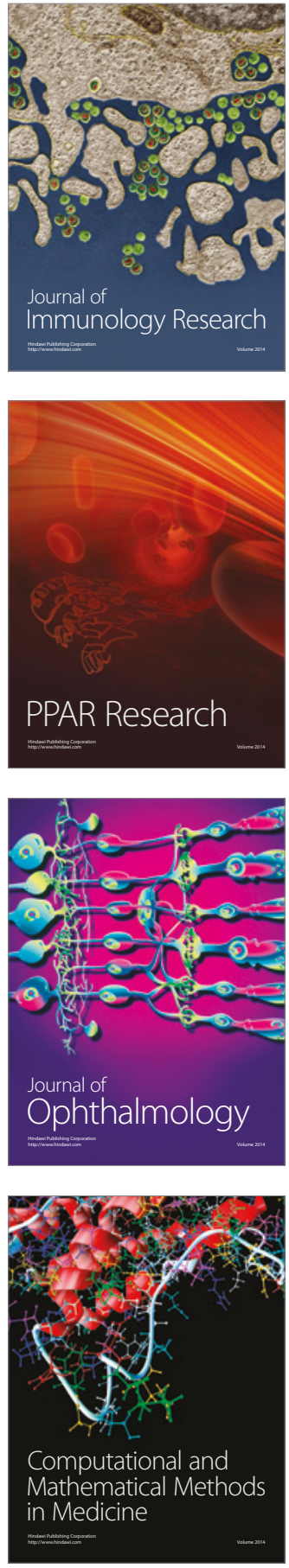

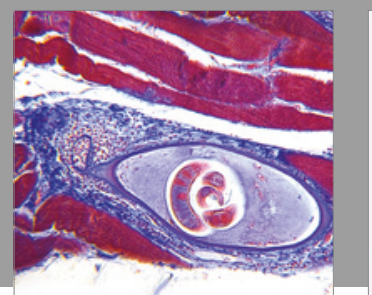

Gastroenterology Research and Practice
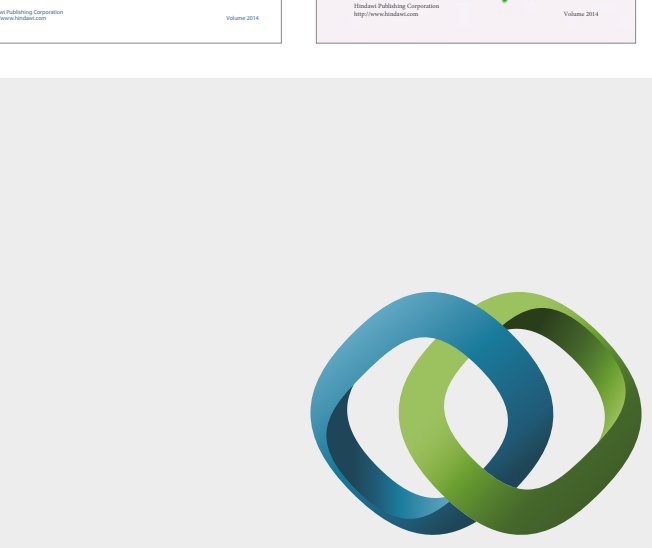

\section{Hindawi}

Submit your manuscripts at

https://www.hindawi.com
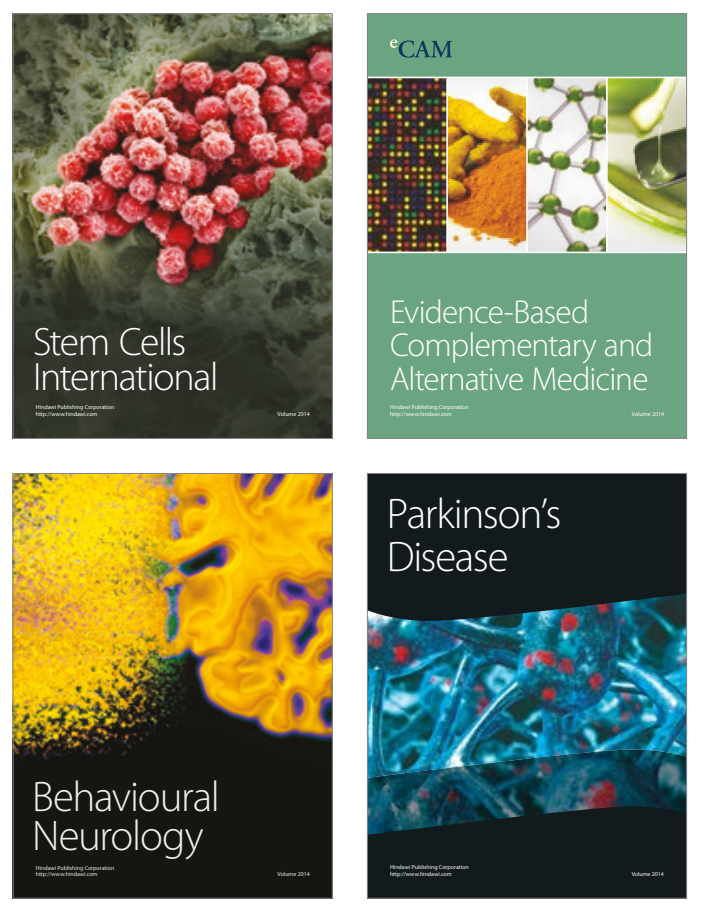
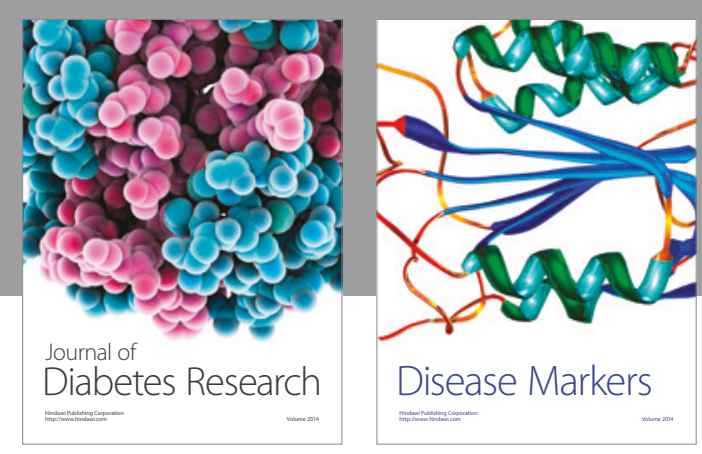

Disease Markers
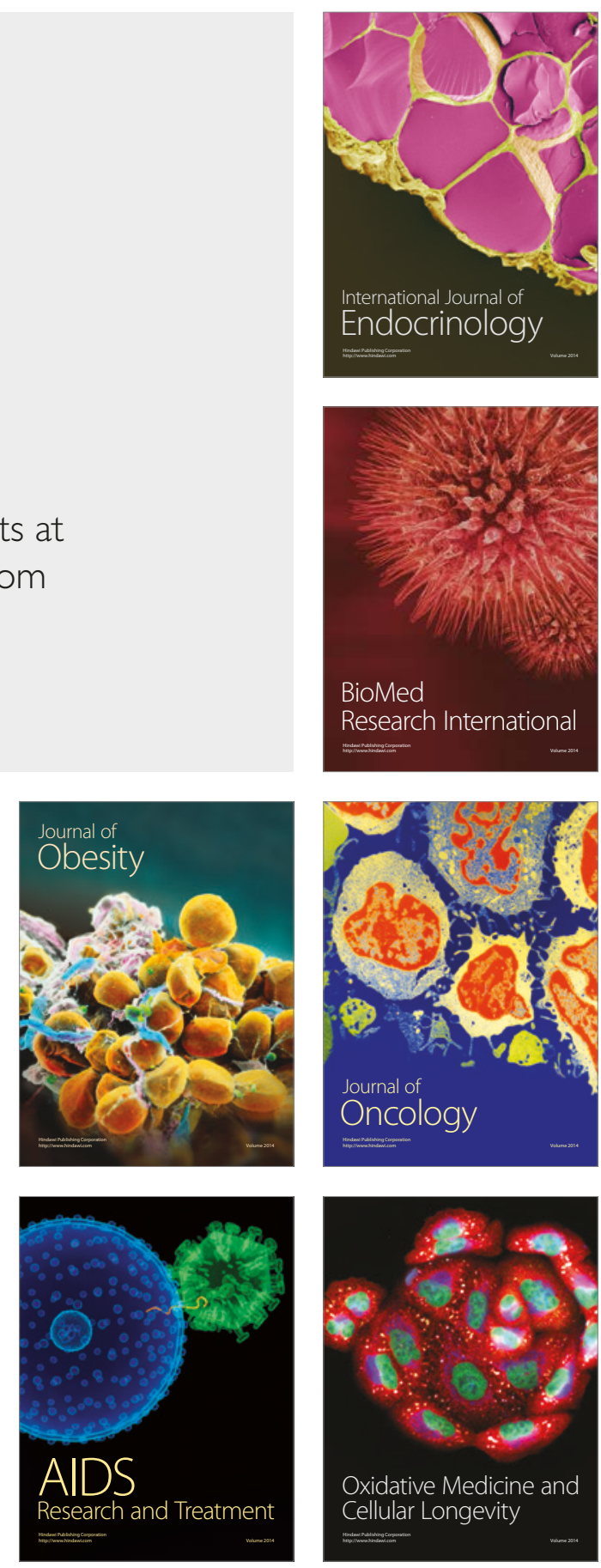